\title{
Neurorestorative Roles of Microgliosis and Astrogliosis in Neuroinflammation and Neurodegeneration
}

\author{
Babatunde Oluwafemi Adetuyi ${ }^{1 *}$, Pere-Ebi Yabrade Toloyai ${ }^{2}$, Evelyn Tarela Ojugbeli ${ }^{2}$, \\ Oyetola Tolulope Oyebanjo ${ }^{3}$, Oluwatosin Adefunke Adetuyi ${ }^{4}$, Chukwuemelie Zedech Uche ${ }^{5}$, \\ Michael Chinedu Olisah ${ }^{6}$, Obinna Chukwuemeka Uchenna Adumanya ${ }^{7}$, Chukwudi Jude \\ Chikwendu $^{8}$, Johra Khan ${ }^{9,10}$, Muhammad Akram ${ }^{11}$, Chinaza Godswill Awuchi ${ }^{12}$, and \\ Chukwuebuka Egbuna ${ }^{8,13}$
}

\footnotetext{
${ }^{1}$ Biochemistry Unit, Department of Natural Sciences, Faculty of Pure and Applied Sciences, Precious Cornerstone University, Ibadan, Nigeria.

${ }^{2}$ Department of Medical Biochemistry, Delta State University, Abraka, Delta State, 330106,

${ }^{3}$ Department of Physiology, Babcock University, Ilishan, Ogun State, 121003.

${ }^{4}$ Department of Chemistry and Biochemistry, Texas Tech University, Lubbock, United State, 79430.

${ }^{5}$ Department of Medical Biochemistry and Molecular Biology, Faculty of Basic Medical Sciences, University of Nigeria, Enugu Campus, Nigeria.

${ }^{6}$ Department of Medical Biochemistry, Faculty of Basic Medical Sciences, Chukwuemeka Odumegwu Ojukwu University, Anambra State- 431124, Nigeria.

${ }^{7}$ Department of Biochemistry, University of Agriculture and Environmental Science, Umuagwo, Imo State, Nigeria.

${ }^{8}$ Department of Biochemistry, Faculty of Natural Sciences, Chukwuemeka Odumegwu Ojukwu University, Anambra State- 431124, Nigeria.

${ }^{9}$ Department of Medical Laboratory Sciences, College of Applied Medical Sciences, Majmaah University, Majmaah, 11952, Saudi Arabia.

${ }^{10}$ Health and Basic Sciences Research Center, Majmaah University, Majmaah, 11952, Saudi Arabia.

${ }^{11}$ Department of Eastern Medicines, Government College University, Faisalabad, Pakistan.

${ }^{12}$ School of Natural and Applied Sciences, Kampala International University, Kampala, Uganda.

${ }^{13}$ Africa Centre of Excellence in Public Health and Toxicological Research (ACE-PUTOR), Nutritional

Biochemistry and Toxicology Unit, University of Port-Harcourt, Rivers State, Nigeria.
}

*Corresponding author: badetuyi@pcu.edu.ng; Phone: +2348162460972

\begin{abstract}
The pathophysiological processes involved in neurodegenerative diseases have not been clearly defined. Nevertheless, a significant aspect of the proof focuses directly on the function of several mechanisms of inflammation. The immune system is represented in the central nervous system by the microglial cell capable of detecting harmful or foreign pathogens, and thus initiates self-activation and neuro-inflammatory processes via phagocytosis and cytokines release, to maintain the cellular microenvironment. Then, microglial cells can spawn an emphasis on persistent inflammation that sometimes precedes or promote the neurodegenerative processes. Hence, the neuro-inflammatory micro-environment turns toxic and damaging to the neuronal cell, leading to degeneration and release of several factors which trigger an inflammatory reaction of the microglia, activating the neurodegenerative cycle. The biomechanical properties of the brain, neuronal regeneration, and plasticity can be modified by reactive gliosis. Defining the inception and development of reactive microgliosis and astrogliosis is vital for better clinical treatments design.
\end{abstract}

Keywords: Microglia, Astrogliosis, Neurodegeneration, Inflammation, Neurorestoration

\section{Article History}

Received: 16 Nov 2021

Accepted: 01 Dec. 2021

Published: 07 Dec. 2021

Scan QR code to view

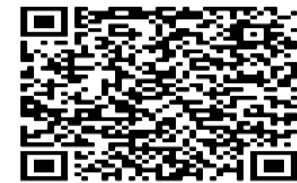

License: CC BY 4.0*

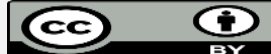

Open Access article.

How to cite this paper: Adetuyi, B. O., Toloyai, P.-E. Y., Ojugbeli, E. T., Adetuyi, O. A., Uche, C. Z., Olisah, M. C., Adumanya, O. C. U., Chikwendu, C. J. Khan, J., Akram, M., Awuchi, C. G., \& Egbuna, C. Neurorestorative Roles of Microgliosis and Astrogliosis in Neuroinflammation and Neurodegeneration. Scicom Journal of Medical and Applied Medical Sciences, 1(1), 1-5. Scicom J Med Appl Med Sci, 1(1), 1-5. https://doi.org/10.54117/sjmams.v1i1.1

- This work is published open access under the Creative Commons Attribution License 4.0, which permits free reuse, remix, redistribution and transformation provided due credit is given. 


\section{List of Abbreviations}

APC: Antigen presenting cells

BBB: Blood brain barrier

CCL: $\quad$ Chemokine (c-c motif) ligand

CCR: Chemokine receptor

CD: $\quad$ Cluster of differentiation

CNS: Central nervous system

CXCL: $\quad c-X-c$ motif chemokine

JAK-STAT: Janus kinase-signal transducer and activator of transcription

MAP-K: Mitogen-activated protein kinase

MCP: Monocyte chemoattractant protein

NFE2L2: Nuclear factor erythroid 2-like-2 protein

NF- $\kappa$ B: Nuclear factor-kappa B

NLRs: NOD-like receptors

NMDA: N-Methyl-D-aspartate

NOD: Nucleotide-binding and oligomerization domain

ORM: Orosomucoid molecule

RNS: $\quad$ Reactive nitrogen Species

ROS: Reactive oxygen species

TGF: Transforming growth factor

TLRs: Toll-like receptors

TNF- $\alpha$ : Tumor necrosis factor alpha

\section{Introduction}

Neurodegeneration is an encompassing word used to define several developments which result in the loss of function, structure, and death of neurons (Fig. 1). Substantial efforts are engaged in clarifying the effect of inflammatory mechanisms in neurodegeneration, which might help in formulating new therapeutics methods aimed at the neuro-immune system to stimulate neuro-restoration. Neuroinflammation is known as a vital biological reaction of the body's tissues to damaging stimuli relating to molecular mediators, blood vessels, and immune cells in the central nervous system (CNS). This process involves movement, accumulation, and attraction of cells supporting a flow of events involved in the impairment and revivification of tissue. In several defects of the central nervous system, the inflammatory response comprises of the activation of the glial cells $[1,2]$

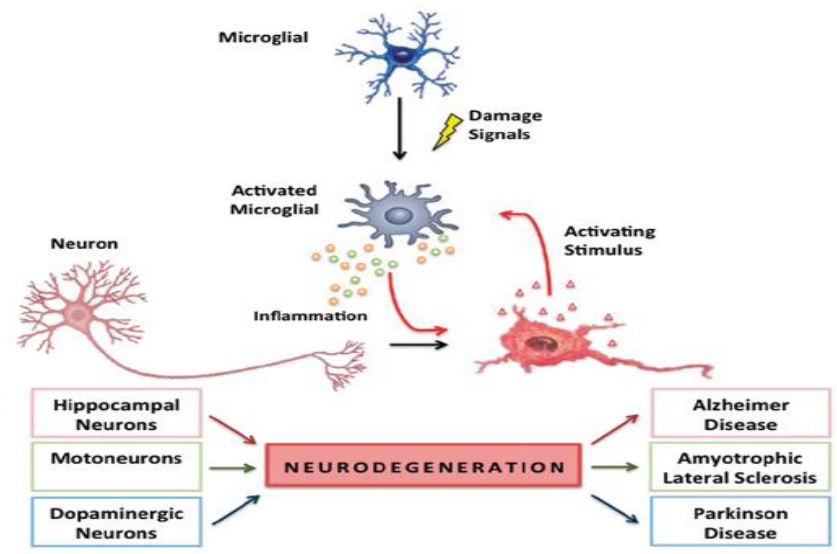

Figure 1: Neuro-inflammation and neuro-degeneration model [5].

The inflammatory responses are prompted to combat infection, moderate injury, and any other stimulus which may include various cell types. The effect of this response depends on several factors released from these cells, possessing self-regulatory capability to repair damaged tissue and eliminate pathogenic elements. However, prolonged response results in chronic inflammation of the environment leading to progressive tissue damage [3]. A shred of important evidence supporting the connection between an increase in age and inflammation is described by an increase in inflammatory mediators for example interleukin (IL)- 6 and -8 and also increase in C-reactive protein in humans with age, with the potential to increase in neurodegenerative diseases and obesity [4].

In the brain, microglia and astrocytes serve as the support of the immune response; to be precise, microglia cells constitute 10 to 15 percent of the brain, which varies regionally and preponderate in the midbrain regions for example the substantia nigra and hippocampus [6]. The blood-brain barrier, parted from the systemic immune system by the immune response of the brain depends on the microglia potential to react as a manifold of immune cells, detect toxins, cytokine levels, injury, and pathogens and also react in a neurotoxic or neurotrophic form akin to the macrophages in the systemic immune system [7].

In a neuro-toxic mode (Fig. 2), activated microglial cells respond to injury and insult by inducing proinflammatory cytokines to attract other astrocytes and microglia in response to bacterial infection and form extensive and diverse factors such as reactive nitrogen species (RNS), reactive oxygen species (ROS), lipid mediators, cytokines and also eliminate cellular wastes as a form of post-infection feedback via phagocytosis [8]. Thus, microglia self-protect against their personal toxic products via chains of antioxidant proteins been moderated by the nuclear factor erythroid 2-like-2 protein (NFE2L2) effects [9]. Microglia are involved in an increasing number of diseases that are central nervous system (CNS)-related; typically stimulated microglia are found in regions of the brain suffering from AIDS-associated Neurodegenerative disease [10].

Astrocytes give neurons functional and structural support, secrete gliotransmitters to control synaptic activity, and get involved in the formation and remodeling of the synapse [11]. They also have the potential to participate and respond to inflammatory in the brain [12]. Though inflammation and immune reaction are regular mechanisms for defense targeted at brain protection from injury, cellular debris, infection, or irregular protein accumulation. In response to several stimuli, activated astrocytes secrete numerous inflammatory and immune mediators for instance anti- and pro-inflammatory chemokines/cytokines that subsequently elicit neurotoxic or neuroprotective effects. In response to immune stimuli, some other glial cell-type of mesodermal origin activated microglia also partake in neurotoxicity and neuroprotection [13] by releasing anti- or pro-inflammatory chemokines/cytokines. These unique forms of microglia activation are known as functional polarization, with involvements in different disorders of the CNS, such as cerebral ischemia and spinal cord injury [14].

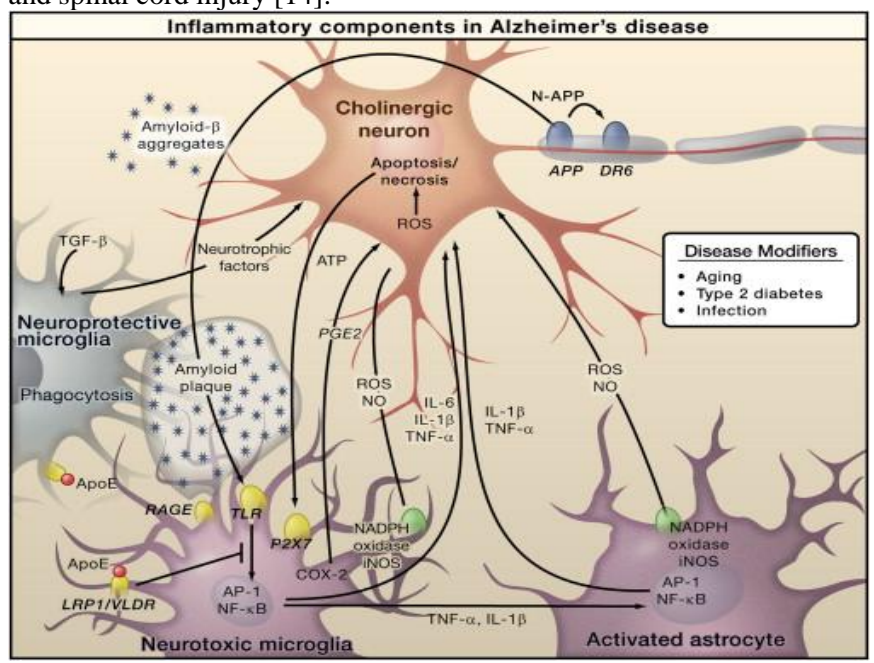

Figure 2: Microglia activation pathology via prolonged systemic inflammation and neurodegenerative cycling [19].

\section{Neuro-inflammation and Microglia}

Microglial cells are located in the spinal cord and brain but specifically in the hippocampus and substantia nigra [15]. These cells are about 5 to 20 percent of the whole populace of glial cells in the CNS and are known to represent the immune system in the CNS, due to their ability to secrete cytotoxic factors, act as antigen-presenting cells (APC), and exert phagocytic effect [16]. They are derivative of macrophages formed in the naive yolk sac via hematopoiesis, then translocate to the emerging neural tube where microglia are developed [17]. In normal conditions, microglia cells guard the brain surroundings by inducing a rapid reaction to alterations and successfully moderate inflammation. Several signs that jeopardize homeostasis of the central nervous system for example residues and/or structures from fungi, viruses, and bacteria; complement factors, cytokines, chemokines, antibodies, and abnormal endogenous 
proteins among others, are detected by the microglial cells and thus initiate their activation [15]. Therefore, there are 2 main functional parts of the microglia-maintenance of the central nervous system and immune defense [17].

Microglial cells possess two functional states of polarization-firstly is the phenotypical polarization to develop a typical pro-inflammatory or a different phenotype that is anti-inflammatory and pro-healing [18] Hence, various anti- and pro-inflammatory cytokines, and other stimuli, can polarize microglia near distinct functional phenotypes.

Inflammatory phenotype of activated cells is described by the up regulation of tumor necrosis factor (TNF)- $\alpha$, CD16 Fc receptors, CD86 CD64, CD32, IL-1 $\beta,-6,-12,-23$, chemokine and inducible nitric oxide synthase (iNOS), however microglia possessing anti-inflammatory phenotype exhibit upregulation of the insulin-like growth factor (IGF)-1, mannose receptor (CD206), arginase (Arg)-1, activating receptor expressed on chitinase 3-like 3 (Ym-1), myeloid cells 2 (TREM2), among others [18].

These factors are embroiled in the stimulation of microglial cells leading to the additional generation of some inflammatory mediators and cytokines, capable of contributing to apoptosis of the neurons in manifold neurodegenerative diseases. With these features, microglial cells are considered as the local immune cells in the brain, and also erogenous to minor changes in central nervous system equilibrium and then readily activated during most neuropathological conditions.

Among other things, a significant point of note is the starting of the disease in which the activated microglial cells begin. For instance, at the early stage of Alzheimer's disease, there is an increase in the activation of microglial; indicating that microglia initiates the removal of dangerous elements contributing to the disease like amyloid- $\beta$ plaques. Hence, an acute neuro-inflammatory response is believed to have a beneficial effect on the central nervous system, with the ability to reduce damage and repair damaged tissue. Besides, microglia have the potential of removing glutamate, which is a recognized neuro-toxic substance that exerts its actions on NMDA (N-Methyl-D-aspartate) receptors from the neurons and then results in the death of the neurons. In Alzheimer's patients, the significance of glutamic acid and related microglia cell role was evidenced by the therapeutic actions of memantine drug (NMDA receptors antagonists) that enhances the cognitive potential and daily functions [20]. Meanwhile, it is imperative to note that microglial cells can be triggered by endogenous proteins or with environmental toxins, resulting in over-activation of cells and generation of RNS and ROS neuronal toxicity [21].

\section{Neuro-inflammation and Astrocytes}

Astrocytes, the fewest abounding and diverse kind of glial cells in the CNS can change their morphology based on their localization, subtype, and developmental stage [22]. For instance, the protoplasmic ones are the gray matter astrocytes, displaying short branches, while the white matte fibrous astrocytes show long unbranched methods [23]. The astrocytes support the components of the neurons in the neural tissue and microglia cell and also respond to insults of all forms in the central nervous system through the reactive astrogliosis; this procedure is sensitive and dependable biomarker of diseased tissues. These cells are accountable for multifaceted and vital functions in a healthy central nervous system, such as their involvement in the primary functions in the processing of information by neural circuits and synaptic transmission [12] and are also involved in synaptogenesis and dynamically control signal transmission, information processing, synaptic and neural plasticity, and also give metabolic support for neurons [24]. As a result, astrocytes have been proven to partake in some essential processes for example regulating environmental homeostasis of ions, $\mathrm{pH}$, the flow of blood, reducing oxidative stress [25], and also accountable for a huge sum of equilibrium tasks in the central nervous system [26]. With these abilities, microglia cells and astrocytes, behave like the core effectors of the neuroinflammatory feedback. After trauma or sense of destruction signal, astrocytic cells together with microglia speedily respond to pathology and undertake significant changes in their functioning and morphology [24] Therefore, the feedback objective is to regulate and eliminate the insult on the brain, but this feedback could have lethal outcomes. Reactive gliosis is a self-sustaining activity that eventually aggravates injury and also signifies a non-physiologic condition in which the astrocytes may forgo their supportive effects [25]. The process that leads to the stimulation of the cells remains uncertain, but several factors tangled in brain damages can stimulate their feedback. For instance, the presence of amyloid in Alzheimer's disease has been confirmed to activate astrocytes. Similar to microglia, astrocytes can also phagocytose and destroy amyloid- $\beta$, and to bring this ability, microglia and astrocytes are triggered via TLRs (Toll-Like Receptors) and RAGE (receptor for advanced glycation end-products), therefore initiating local inflammation [27]. Once the astrocytes response is triggered, there is a change in their morphology and significantly intensifies the expression of the glial fibrillary acidic protein (GFAP), which is a known indicator of astrocytes reactivity [28]. The changes result in a disorder in the astrocyte's normal activities, which are important for the normal function of the neurons. Internally, astrocytes activation includes stimulation of transcription factor- $N F-\kappa B$, regulating the release of adhesion molecules and chemokine, and hence favors outer lymphocyte penetration and upregulating inflammatory response resulting in neurodegeneration [27]. Besides, the transcriptional activity of NF- $\mathrm{B}$ inhibition in astrocytes can broadly decrease inflammation, therefore signifying $N F-\kappa B$ inhibition in the astrocytes as a possible therapy for diseases like Alzheimer's disease [30].

With this evidence, it has been confirmed that activated astrocytes have the potential to initiate neurodegeneration; furthermore, activated astrocytes display inflammation-related factors, for example, S100 $\beta$ peptide, which represent a key factor for neuro-inflammation. The S10 $\beta$ protein, a neurotrophin produced by astrocytes, is responsible for the development, function of neurons, and survival under physiological conditions. The peptide $\mathrm{S} 100 \beta$ is over-expressed and is identified with the pathology progression of subjects with severe brain trauma and neurodegenerative diseases [27]

\section{Crosstalk between Astrocyte and Microglia}

Microglia, the major immune cells in the CNS, partake in many neuropathological conditions and, in synergy with astrocytes, aid the recovery of the CNS from injury and stress [31]. Due to their bi-directional conversation and autocrine feedback, during the injury modulation in the CNS, the cross-talk amid astrocytes and microglia has come into the vanguard of glial research and electromagnetic force therapy utilized in inducing neuro-restoration. At the epicenter of this mutual adjustment is the control of microglial cells of the innate immune roles of astrocytes, influencing their role, either neuro-toxic or neuroprotective [31]. Microglia, activated before astrocytes, release NADPH oxidase derived hydrogen peroxide $\left(\mathrm{H}_{2} \mathrm{O}_{2}\right)$ [32], complement component $1 \mathrm{q}(\mathrm{C} 1 \mathrm{q})$, tumor necrosis factor (TNF)- $\alpha$, and interleukin (IL)-1 $\alpha$ (Fig. 3) [33] to control astrocyte stimulation and promote $\mathrm{A} 1$ reactive astrocytosis which is the major destructive mechanism of astrocytes activity.

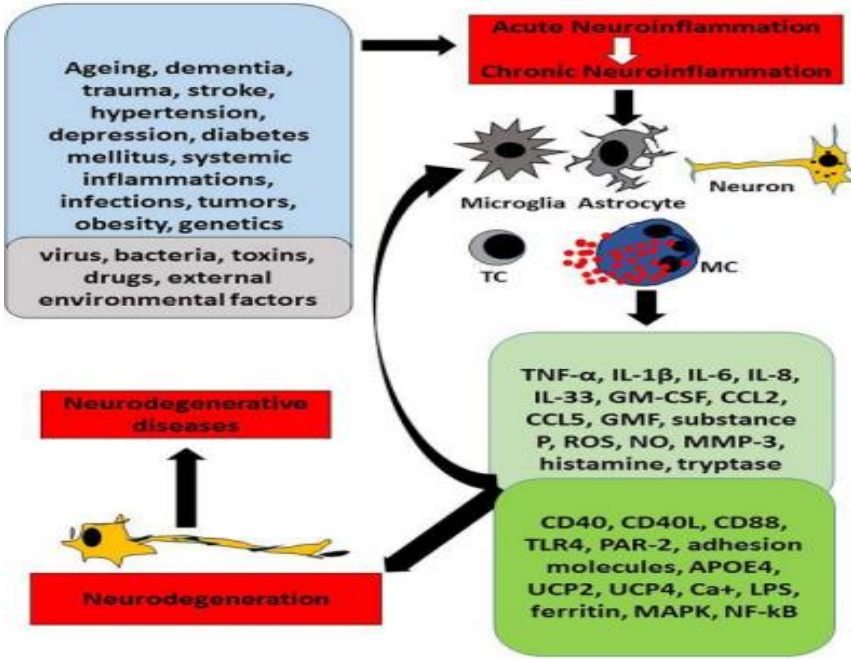

Figure 3: Schematic diagram showing Neuro-inflammation-mediated neurodegeneration in the brain [34].

This enables the microglial cell the primary target for electromagnetic force (EMF) therapy of the central nervous system. Though astrocytic 
cells are known as neuronal supportive cells, helping with the regulation of the CNS homeostasis, several studies describe astrocytes' function in the microglial functions and phenotypes regulation, and the inborn immune response in the CNS [35]. Within the astrocyte activation cascade, is a major signaling molecule which is orosomucoid-2 (ORM2), having a very important function in the release of the proinflammatory cytokine, and with the potential to control the infiltration of immune cells via the blood-brain barrier (BBB), enables astrocytes to be functioning actor in neuro-inflammation and subsequently in neurorestoration. Based on the state of the stimulus, it can stimulate tissue repair (A2 reactive astrocytosis) and regeneration or increase the immune response causing more tissue damage (A1 reactive astrocytosis) [36].

With the release of orosomucoid-2 (ORM-2), which inhibit chemokine (C-C) motif ligand-4 and chemokine receptor type 5 (CCL4-CCR5) interaction, astrocytes can either hinder activation of the microglia and release pro-inflammatory cytokine or improve the activity of the microglia by upregulating lipocalin (LCN)-2, monocyte chemoattractant protein (MCP)-1/chemokine ligand (CCL)-2, Interferon $\gamma$-induced protein (IP) $10 / \mathrm{c}-\mathrm{x}$-c-motif chemokine (CXCL)-10, or transforming growth factor (TGF)- $\beta$ [4]. Besides, by showing innate immune pattern recognition receptors (PRRs), for example, scavenger receptors, complement, mannose, NOD-like (NLRs), and Toll-like (TLRs), astrocytes institute a close cross-talk with the neighboring microglia in the CNS for removal of pathogens, repair the tissue, or scar formation induction [37]. Likewise, recent studies have established the astrocytemicroglia cross-talk is an important step in the innate reaction to injury or inflammation in the CNS, demonstrating that the astrocyte's response to toll-like receptor (TLR)-2, 3, and 4 is significantly boosted by or directly associated to, the existence of microglial cells within nearby tissue [38] This shows an important interaction between the microglia and astrocytes in neuro-repair and neuro-restoration.

Simply through cautiously controlled interactions of astrocytes and microglia that reaction to inflammation can be resolved and controlled. A1 astrocytic cells are pro-inflammatory, showing gene upregulation possibly damaging to synapses, and are initiated by microglia release of interleukin (IL)- $\alpha, \mathrm{TNF} \alpha$, and complement component $(\mathrm{C} 1 \mathrm{q}), \mathrm{A} 2$ reactive astrocytes release proteins that stimulate synaptogenesis of the CNS [18] Also, as are triggered under ischemic states; A2 astrocytes have neurorestorative and -protective effects and display the phenotypes required to be induced for electromagnetic force (EMF) therapy to success leads to neuro-regeneration [38].

Working synergistically with astrocytic cells, the highly abounding cells in the CNS, microglia -typically function as chaperones to the astrocytes, controlling astrocyte's innate immune effects at pathological states by secreting molecules having an impact on the intracellular signal transduction via MAPK and JAK-STAT pathways. To further increase the inflammatory response, microglial cells upregulate the nuclear factor kappa B (NF- $\mathrm{B}$ ) signaling pathway; and once triggered, astrocytes augment pro-inflammatory gene expression, and promote the formation of pro-inflammatory growth factors, chemokines, and cytokines. Then, astrocytes control microglial cell phenotypes and functions via the astrocyte-derived factors, complemented proteins, cytokines, and chemokines [15].

\section{Conclusion}

The origin and various causes of CNS microglia and astrocytes activation, amplification, and termination are vital in unraveling treatment for a wellmodulated and maintained systemic immune system response. Seeing it as a possible therapeutic step, inhibiting the inflammatory process is a potential therapeutic strategy to combat inflammation-induced neurodegeneration.

\section{References}

[1] Jauchem J.R. Effects of low-level radio-frequency ( $3 \mathrm{kHz}$ to $300 \mathrm{GHz}$ ) energy on human cardiovascular, reproductive, immune, and other systems: a review of the recent literature. International journal of hygiene and environmental health, 2008, 211, 1-29. https://doi.org/10.1016/j.ijheh.2007.05.001.

[2] Rosado, M.M.; Simkó, M.; Mattsson, M.O.; Pioli, C. Immunemodulating perspectives for low frequency electromagnetic fields in innate immunity. Frontiers in public health, 2018, 6, 85-94. https://doi.org/10.3389/fpubh.2018.00085.

[3] Howcroft T.K.; Campisi J.; Louis G.B.; Smith M.T.; Wise B.; WyssCoray $\mathrm{T}$. The role of inflammation in age-related disease. Aging (Albany NY), 2013, 5, 84-99. https://doi.org/10.18632/aging.100531.

[4] Norden, D.M.; Fenn, A. M.; Dugan, A.; Godbout, J. P. TGF $\beta$ produced by IL-10 redirected astrocytes attenuates microglial activation. Glia, 2014, 62, 881-895. https://doi.org/10.1002/glia.22647.

[5] Inelia Morales, Gonzalo A. Farias, Nicole Cortes and Ricardo B. Maccioni (2016) Neuroinflammation and Neurodegeneration doi:10.5772/64545. https://doi.org/10.5772/64545.

[6] Block, M.L.; Zecca, L.; Hong, J.S. "Microglia-mediated neurotoxicity: uncovering the molecular mechanisms. Nature Reviews Neuroscience, 2007, 8, 57-69. https://doi.org/10.1038/nrn2038.

[7] Colton, C.A. Heterogeneity of microglial activation in the innate immune response in the brain. Journal of neuroimmune pharmacology, 2009, 4, 399-418. https://doi.org/10.1007/s11481009-9164-4.

[8] Colton, C.A.; Wilcock, D.M. Assessing activation states in microglia. CNS \& Neurological Disorders-Drug Targets (Formerly Current Drug Targets-CNS \& Neurological Disorders), 2010, 9,174-191. https://doi.org/10.2174/187152710791012053.

[9] de Vries, H.E.; Witte, M.; Hondius, D.; Rozemuller, A.J.; Drukarch, B.; Hoozemans, J. Nrf2-induced antioxidant protection: a promising target to counteract ROS-mediated damage in neurodegenerative disease? Free Radical Biology and Medicine, 2008, 45, 1375-1381. https://doi.org/10.1016/j.freeradbiomed.2008.09.001.

[10] Qin, L.; Crews, F.T. NADPH oxidase and reactive oxygen species contribute to alcohol-induced microglial activation and neurodegeneration. Journal of neuroinflammation, 2012, 9, 1-19. https://doi.org/10.1186/1742-2094-9-5.

[11] Perea, G.; Navarrete, M.; Araque, A. Tripartite synapses: astrocytes process and control synaptic information. Trends in neurosciences, 2009, 32, 421-431. https://doi.org/10.1016/j.tins.2009.05.001.

[12] Sofroniew, M.V.; Vinters, H.V. Astrocytes: biology and pathology. Acta neuropathologica, 2010, 119, 7-35. https://doi.org/10.1007/s00401-009-0619-8.

[13] Farina, C.; Aloisi, F.; Meinl, E. Astrocytes are active players in cerebral innate immunity. Trends in immunology, 2007, 28, 138145. https://doi.org/10.1016/j.it.2007.01.005.

[14] Mandrekar-Colucci, S.; Karlo, J.C.; Landreth, G. E. Mechanisms underlying the rapid peroxisome proliferator-activated receptor- $\gamma$ mediated amyloid clearance and reversal of cognitive deficits in a murine model of Alzheimer's disease. Journal of Neuroscience, 2012, 32, 10117-10128. https://doi.org/10.1523/JNEUROSCI.5268-11.2012.

[15] Venneti, S.; Wiley, C.A.; Kofler, J. Imaging microglial activation during neuroinflammation and Alzheimer's disease. Journal of Neuroimmune Pharmacology, 2009, 4, 227-243. https://doi.org/10.1007/s11481-008-9142-2.

[16] Hanisch U.K.; Kettenmann, H. Microglia: active sensor and versatile effector cells in the normal and pathologic brain. Nature neuroscience, 2007, 10, 1387-1394. https://doi.org/10.1038/nn1997.

[17] Ginhoux, F.; Lim, S.; Hoeffel, G.; Low, D.; Huber, T. Origin and differentiation of microglia. Frontiers in cellular neuroscience, 2013, 7, 45-54. https://doi.org/10.3389/fncel.2013.00045.

[18] Jha, M. K.; Lee, W. H.; Suk, K. Functional polarization of neuroglia: implications in neuroinflammation and neurological disorders. Biochemical pharmacology, 2016, 103, 1-16.

https://doi.org/10.1016/j.bcp.2015.11.003.

[19] Christopher K. Glass, Kaoru Saijo, Beate Winner, Maria Carolins Marchetto, Fred H. Gage (2010) Mechanisms underlying inflammation in Neurodegeneration, (40)6, pp918-934. https://doi.org/10.1016/j.cell.2010.02.016.

[20] Mrak, R.E. Microglia in Alzheimer brain: a neuropathological perspective. International journal of Alzheimer's disease, 2012, 12, 342-354. https://doi.org/10.1155/2012/165021.

[21] Innamorato, N.G.; Lastres-Becker, I.; Cuadrado, A. Role of microglial redox balance in modulation of neuroinflammation. Current opinion in neurology, 2009, 22, 308-314. https://doi.org/10.1097/WCO.0b013e32832a3225. 
[22] Bronzuoli, M.R.; Iacomino, A.; Esposito, G.; Steardo, L.; Scuderi, C. Does neuroinflammation turn on the flame in Alzheimer's disease? Focus on astrocytes. Frontiers in neuroscience, 2015, 9 , 259-267. https://doi.org/10.3389/fnins.2015.00259.

[23] Verkhratsky, A.; Parpura, V. Astrogliopathology in neurological, neurodevelopmental and psychiatric disorders. Neurobiology of disease, 2016, 85, 254-261. https://doi.org/10.1016/j.nbd.2015.03.025.

[24] Sofroniew, M.V. Multiple roles for astrocytes as effectors of cytokines and inflammatory mediators. The Neuroscientist, 2014, 20, 160-172. https://doi.org/10.1177/1073858413504466.

[25] Hertz, L. Book review: Glial physiology and pathophysiology by Alexei Verkhratsky and Arthur Butt, Wiley-Blackwell, 2013. Frontiers in Systems Neuroscience, 2014, 8, 17-27. https://doi.org/10.3389/fnsys.2014.00017.

[26] Merazrios, M.A.; Toral-Rios, D.; Franco-Bocanegra, D.; VilledaHernández, J.; Campos-Peña, V. Inflammatory process in Alzheimer's Disease. Frontiers in integrative neuroscience, 2013, 7, 59-67. https://doi.org/10.3389/fnint.2013.00059.

[27] Chow, S.K.; Yu, D.; MacDonald, C.L.; Buibas, M.; Silva, G.A. Amyloid $\beta$-peptide directly induces spontaneous calcium transients, delayed intercellular calcium waves and gliosis in rat cortical astrocytes. ASN neuro, 2009, 2, 235-246. https://doi.org/10.1042/AN20090035.

[28] Medeiros R.; LaFerla, F.M. Astrocytes: conductors of the Alzheimer disease neuroinflammatory symphony. Experimental neurology, 2013, 239, 133-138. https://doi.org/10.1016/j.expneurol.2012.10.007.

[29] Jha, M.K.; Jo, M.; Kim, J.H.; Suk, K. Microglia-astrocyte crosstalk: an intimate molecular conversation. The Neuroscientist, 2019, 25, 227-240. https://doi.org/10.1177/1073858418783959.

[30] Hou, L.; Zhou, X.; Zhang, C.; Wang, K.; Liu, X.; Che,Y. NADPH oxidase-derived $\mathrm{H} 2 \mathrm{O} 2$ mediates the regulatory effects of microglia on astrogliosis in experimental models of Parkinson's disease. Redox biology, 2017, 12, 162-170.

https://doi.org/10.1016/j.redox.2017.02.016

[31] Liddelow, S.A.; Guttenplan, K. A.; Clarke, L. E.; Bennett, F. C.; Bohlen, C. J.; Schirmer, L. Neurotoxic reactive astrocytes are induced by activated microglia. Nature, 2017, 541, 481-487. https://doi.org/10.1038/nature21029.

[32] Barrientos, R.; Kitt, M.; Watkins, L.; Maier, S. "Neuroinflammation in the normal aging hippocampus. Neuroscience, 2015, 309, 8499. https://doi.org/10.1016/j.neuroscience.2015.03.007.

[33] Klein, R. S.; Hunter, C. A. Protective and pathological immunity during central nervous system infections. Immunity, 2017, 46, 891-909. https://doi.org/10.1016/j.immuni.2017.06.012.

[34] Jang, E.; Kim, J.H.; Lee, S.; Kim, J.H.; Seo, J.W.; Jin, M. Phenotypic polarization of activated astrocytes: the critical role of lipocalin-2 in the classical inflammatory activation of astrocytes. The Journal of Immunology, 2013, 191, 5204-5219. https://doi.org/10.4049/jimmunol.1301637.

[35] Tian, L.; Ma, L.; Kaarela, T.; Li, Z. Neuroimmune crosstalk in the central nervous system and its significance for neurological diseases. Journal of neuroinflammation, 2012, 9, 1-10. https://doi.org/10.1186/1742-2094-9-155.

[36] Holm, T.H.; Draeby, D.; Owens, T. Microglia are required for astroglial Toll-like receptor 4 response and for optimal TLR2 and TLR3 response. Glia, 2012, 60, 630-638. https://doi.org/10.1002/glia.22296.

[37] Pascual, O.; Achour, S.B.; Rostaing, P.; Triller, A.; Bessis, A. Microglia activation triggers astrocyte-mediated modulation of excitatory neurotransmission. Proceedings of the National Academy of Sciences, 2012, 109, 197-205. https://doi.org/10.1073/pnas.1111098109.

- Thank you for publishing with us. 